\title{
Double fibres and double covers: paucity of rational points
}

by

\author{
J.-L. Colliot-Thélène (Orsay),
}

A. N. Skorobogatov (Moscow and Marseille) and Sir Peter Swinnerton-Dyer (Cambridge)

\section{J. W. S. Cassels die natali oblatum}

Introduction. Let $X / k$ be a smooth, projective, geometrically connected variety over a number field $k$. Assume that we are given a proper flat morphism $p: X \rightarrow \mathbb{P}_{k}^{1}$ with (smooth) geometrically integral generic fibre.

In a series of recent papers ([R], [CT/SwD], [SwD], [Sk], [CT/Sk/SwD.1], $[\mathrm{CT} / \mathrm{Sk} / \mathrm{SwD} .2]$ ), under specific assumptions on the pencil, various authors have investigated the Zariski density of the set $X(k)$ of $k$-rational points and its topological density with respect to some embedding $X(k) \subset X\left(k_{v}\right)$, where $k_{v}$ denotes a completion of $k$.

In these various papers, the assumptions made imply that no fibre of $p$ is multiple. In the present paper, we go the other way. We look at pencils which possess multiple fibres, and we investigate the consequences for the rational points of the total space $X$. In the interest of simplicity, we restrict attention to fibres which are double. A fibre $X_{P}=p^{-1}(P)$ over a closed point $P \in \mathbb{P}_{k}^{1}$ is called double if as a divisor on $X$ it is a double.

Here are the main results:

1. If there are at least 5 geometric double fibres, then the $k$-rational points are not dense for the Zariski topology on $X$; more precisely, they lie in the union of finitely many fibres of $p$.

2. This easily leads to examples of surfaces over a number field which do not dominate any variety of general type, but which nevertheless do not acquire Zariski-dense $K$-rational points over any finite extension $K$ of the ground field (question raised by J. Harris).

3. We give examples of (connected, smooth, projective) surfaces $X$ over the rationals $\mathbb{Q}$, whose set of rational points $X(\mathbb{Q})$ is dense for the Zariski topology on $X$, and indeed is topologically dense in one connected com- 
ponent of the set $X(\mathbb{R})$ of real points (equipped with the real topology), whereas the closure of $X(\mathbb{Q})$ in $X(\mathbb{R})$ (for that topology) is not the union of finitely many components of $X(\mathbb{R})$, thereby contradicting a conjecture of B. Mazur.

\section{Unramified double covers}

Proposition 1.1. Let $k$ be a field, $\operatorname{char}(k) \neq 2$, and let $X / k$ be $a$ smooth, integral $k$-variety. Let $k(X)$ be its function field. The natural map $H_{\text {ét }}^{1}(X, \mathbb{Z} / 2) \rightarrow H_{\text {ét }}^{1}(k(X), \mathbb{Z} / 2)=k(X)^{*} / k(X)^{* 2}$ of étale cohomology groups induces a bijection between the group of isomorphism classes of $\mathbb{Z} / 2$-torsors over $X$ (which coincides with $H_{\text {èt }}^{1}(X, \mathbb{Z} / 2)$ ) and the subgroup of $k(X)^{*} / k(X)^{* 2}$ consisting of classes of functions whose divisor on $X$ is a double. Let $\mathcal{T} / X$ be a $\mathbb{Z} / 2$-torsor, and let $f \in k(X)^{*}$ correspond to $\mathcal{T}$ under the above map. Let $V \subset X$ be an open set where $f$ is invertible. Then the restriction $\mathcal{T}_{V} / V$ of $\mathcal{T}$ to $V$ is isomorphic to the $\mathbb{Z} / 2$-torsor over $V$ given as the fibre product $V \times_{\mathbb{G}_{m}} \mathbb{G}_{m}$, where $V \rightarrow \mathbb{G}_{m, k}$ is given by $f$, and $\mathbb{G}_{m} \rightarrow \mathbb{G}_{m}$ is given by $x \mapsto x^{2}$.

Informally speaking, $\mathcal{T}_{V}$ is given by the equation $f=u^{2}$ over $V$.

Proof. This is well known, and may be viewed as a very special case of [CT/S.2], Thm. 2.3.1, p. 421. A direct proof runs as follows. Let $\mathcal{T} / X$ be a $\mathbb{Z} / 2$-torsor. If the projection $q: \mathcal{T} \rightarrow X$ has a section at the generic point of $X$, then since $q$ is a finite morphism, it has a section over an open set which contains all codimension one points of $X$. Since $X$ is normal, such a section extends to a section of $q$. Thus the map $H_{\text {êt }}^{1}(X, \mathbb{Z} / 2) \rightarrow H_{\text {êt }}^{1}(k(X), \mathbb{Z} / 2)$ is injective. Using the Kummer sequence

$$
1 \rightarrow \mathbb{Z} / 2 \rightarrow \mathbb{G}_{m} \stackrel{x \mapsto x^{2}}{\longrightarrow} \mathbb{G}_{m} \rightarrow 1
$$

and the triviality of $H_{\text {ét }}^{1}\left(A, \mathbb{G}_{m}\right)$ for $A$ a local ring, one then completes the proof of the proposition.

Given a $k$-variety and a $\mathbb{Z} / 2$-torsor $q: \mathcal{T} \rightarrow X$ over $X$, we have the evaluation map $\theta=\theta_{\mathcal{T}}: X(k) \rightarrow H_{\text {ét }}^{1}(k, \mathbb{Z} / 2)=k^{*} / k^{* 2}$. This evaluation map induces a decomposition of the set $X(k)$ of $k$-rational points:

$$
X(k)=\bigcup_{\alpha \in \operatorname{Im} \theta} \theta_{\mathcal{T}}^{-1}(\alpha),
$$

which may be rewritten as

$$
X(k)=\bigcup_{\alpha \in \operatorname{Im} \theta} q_{\alpha}\left(\mathcal{T}^{\alpha}(k)\right)
$$

where $q_{\alpha}: \mathcal{T}^{\alpha} \rightarrow X$ denotes a $\mathbb{Z} / 2$-torsor with class $[\mathcal{T}]-\alpha$ in $H_{\text {ét }}^{1}(X, \mathbb{Z} / 2)$ ([CT/S.2], (2.7.2), p. 443). For $V$ and $f \in k[V]^{*}$ as in Proposition 1.1, and 
$a \in k^{*}$ a representative of $\alpha \in k^{*} / k^{* 2}$, the restriction of $\mathcal{T}^{\alpha}$ over $V$ is isomorphic to the $\mathbb{Z} / 2$-torsor given by $f=a u^{2}$.

Proposition 1.2. Let $k$ be a field finitely generated over $\mathbb{Q}$, let $X / k$ be an integral, smooth, proper $k$-variety. Let $\mathcal{T} / X$ be a $\mathbb{Z} / 2$-torsor. Then the image of the evaluation map $\theta_{\mathcal{T}}: X(k) \rightarrow H_{\text {ét }}^{1}(k, \mathbb{Z} / 2)=k^{*} / k^{* 2}$ is finite. With notation as above, this induces a finite decomposition

$$
X(k)=\bigcup_{\alpha \in \operatorname{Im} \theta} q_{\alpha}\left(\mathcal{T}^{\alpha}(k)\right)
$$

of the set of rational points of $X$.

Pr o of. This is well known for $k$ a number field. The theorem holds with $\mathbb{Z} / 2$ replaced by an arbitrary $k$-group of multiplicative type (see [CT/S.1], Prop. 2, and [CT/S.2], Théorème 2.7.3, p. 444). The proof may be phrased in a number of ways, for instance as an application of Weil's theory of distributions [W.2], which formalizes some of the arguments in the weak Mordell-Weil theorem. We shall give a proof in this spirit in a more complicated situation in Theorem 2.3 below. That the result holds over a field finitely generated over $\mathbb{Q}$ is also well known (this uses the finiteness theorems of Severi, Néron and Roquette).

Let $k$ be a number field. Decompositions

$$
X(k)=\bigcup_{\alpha \in \operatorname{Im} \theta} q_{\alpha}\left(\mathcal{T}^{\alpha}(k)\right)
$$

as in the above proposition are usually referred to as "descents". Namely, the study of the set of rational points on $X$ is reduced to the study of rational points on the finite number of auxiliary varieties $\mathcal{T}^{\alpha}$ (see [Ca.2], [CT/S.2]). This may be used in a number of ways:

1. To discuss the very existence of $k$-rational points on $X$, from the point of view of the Hasse principle. Let $k_{v}$ denote the completion of $k$ at the place $v$. It may happen that for each place $v$ of $k$, the set $X\left(k_{v}\right)$ is not empty, but nevertheless that $X(k)$ is empty. Such is the case if for each auxiliary $\mathcal{T}^{\alpha}$ there exists a place $v$ of $k$ with $\mathcal{T}^{\alpha}\left(k_{v}\right)=\emptyset$. It is quite likely that such an obstruction to the Hasse principle may be accounted for by the Brauer-Manin obstruction (cf. [CT/S.2], Chap. 3).

2. To discuss the Zariski density of $X(k)$ in $X$. The problem may turn out to be easier on the auxiliary varieties than on the original $X$. In some of our earlier work on rational varieties, specific descents led to examples where it was obvious that $\mathcal{T}^{\alpha}(k)$ was Zariski-dense on $\mathcal{T}^{\alpha}$ whenever it was not empty, thereby yielding the analogous result for $X$. Further below, we shall go the other way round and give examples where $k$-points are not Zariski-dense on any of the $\mathcal{T}^{\alpha}$. In the situation under consideration here, 
where the projection maps $\mathcal{T}^{\alpha} \rightarrow X$ are finite, Proposition 1.2 implies that $X(k)$ is not Zariski-dense in $X$ (see Section 3).

3. To discuss density properties of $X(k)$ in finite products $\prod_{v \in S} X\left(k_{v}\right)$, where $S$ denotes a finite set of places of $k$ and each $X\left(k_{v}\right)$ is equipped with the topology coming from $k_{v}$. Here we shall restrict the discussion to the very specific case where $k=\mathbb{Q}$ and $S$ consists of one place, the real place. One then asks for a description of the topological closure of $X(\mathbb{Q})$ in $X(\mathbb{R})$.

In the situation described above, we have the clearly finite decomposition

$$
X(\mathbb{R})=\bigcup_{\alpha \in \operatorname{Im} \theta_{\mathbb{R}}} q_{\alpha}\left(\mathcal{T}^{\alpha}(\mathbb{R})\right)
$$

as well as the finite decomposition

$$
X(\mathbb{Q})=\bigcup_{\alpha \in \operatorname{Im} \theta_{\mathbb{Q}}} q_{\alpha}\left(\mathcal{T}^{\alpha}(\mathbb{Q})\right) .
$$

Since the map $\theta_{\mathbb{R}}: X(\mathbb{R}) \rightarrow \mathbb{R}^{*} / \mathbb{R}^{* 2}$ is continuous, each $q_{\alpha}\left(\mathcal{T}^{\alpha}(\mathbb{R})\right)=\theta^{-1}(\alpha)$ is open. We thus get a decomposition of $X(\mathbb{R})$ into a disjoint union of two open sets $X(\mathbb{R})_{+}$and $X(\mathbb{R})_{-}$(some of them possibly empty), each of which is a union of connected components of $X(\mathbb{R})$. Since the decompositions over $\mathbb{Q}$ and over $\mathbb{R}$ are compatible, we see that the set of $\mathbb{Q}$-points lying in $X(\mathbb{R})_{+}$ admits the finite disjoint decomposition

$$
X(\mathbb{Q})_{+}=\bigcup_{\alpha \in \operatorname{Im} \theta_{\mathbb{Q}}, \alpha>0} q_{\alpha}\left(\mathcal{T}^{\alpha}(\mathbb{Q})\right)
$$

whereas the set of $\mathbb{Q}$-points lying in $X(\mathbb{R})_{\text {- admits the finite disjoint de- }}$ composition

$$
X(\mathbb{Q})_{-}=\bigcup_{\alpha \in \operatorname{Im} \theta_{\mathbb{Q}}, \alpha<0} q_{\alpha}\left(\mathcal{T}^{\alpha}(\mathbb{Q})\right) .
$$

Now the description of the topological closure of the set of $\mathbb{Q}$-points may be easier to handle on the $\mathcal{T}^{\alpha}$ than on $X$ itself. Suppose that the following three conditions hold:

(a) There exists $\alpha \in \operatorname{Im} \theta_{\mathbb{Q}}, \alpha>0$, such that $\mathcal{T}^{\alpha}(\mathbb{Q})$ is Zariski-dense in $\mathcal{T}^{\alpha}$.

(b) There exists $\alpha \in \operatorname{Im} \theta_{\mathbb{Q}}, \alpha<0$, such that $\mathcal{T}^{\alpha}(\mathbb{Q}) \neq \emptyset$.

(c) For all $\alpha \in \operatorname{Im} \theta_{\mathbb{Q}}, \alpha<0$, the set $\mathcal{T}^{\alpha}(\mathbb{Q})$ is not Zariski-dense in $\mathcal{T}^{\alpha}$.

Then $X(\mathbb{Q})$ is Zariski-dense in $X$, but the topological closure of $X(\mathbb{Q})$ in $X(\mathbb{R})$ does not consist of a union of connected components of $X(\mathbb{R})$, thereby contradicting a conjecture of B. Mazur [Maz.1]. Actual examples will be constructed in Section 5 . 
2. Double fibres. Let $k$ be a field. In this paper, we shall say that a fibration $p: X \rightarrow \mathbb{P}_{k}^{1}$ over the projective line is standard if it has the following properties: The $k$-variety $X$ is geometrically integral, smooth and projective; the map $p: X \rightarrow \mathbb{P}_{k}^{1}$ is a surjective morphism whose (smooth) generic fibre is geometrically integral.

Given a closed point $P \in \mathbb{P}_{k}^{1}$, with residue field $k(P)$, we denote by $X_{P}$ the fibre of $p$ above $P$. This is a $k(P)$-scheme. It also defines a divisor $\left[X_{P}\right]$ on the variety $X$. The fibre $X_{P}$ will be called a double fibre if the divisor $\left[X_{P}\right]$ is the double of a divisor on $X$ : there exists a divisor $\Delta_{P}$ on $X$ such that $\left[X_{P}\right]=2 \Delta_{P}$. The divisor $\Delta_{P}$ is effective, but we do not demand that it be irreducible or reduced (multiplicity free).

Theorem 2.1. Let $k$ be a field finitely generated over $\mathbb{Q}$. Let $p: X \rightarrow \mathbb{P}_{k}^{1}$ be a standard fibration. Let $f \in k\left(\mathbb{P}^{1}\right)^{*}$ be a rational function. Assume that for each closed point $P$ of $\mathbb{P}^{1}$ where the order of $f$ is odd, the fibre $X_{P}$ is a double fibre. Given $\alpha \in k^{*} / k^{* 2}$, let $a \in k^{*}$ be a lift of $\alpha$, and let $C^{\alpha} / \mathbb{P}_{k}^{1}$ denote the smooth projective curve which is $k$-birational to the double cover of $\mathbb{P}^{1}$ with equation $f=a u^{2}$. Then there exists a finite set $I \subset k^{*} / k^{* 2}$ of $\mathbb{Z} / 2$-torsors $q_{\alpha}: \mathcal{T}^{\alpha} \rightarrow X$, each equipped with a dominant $k$-rational map $\mathcal{T}^{\alpha} \rightarrow C^{\alpha}$, and a finite decomposition

$$
X(k)=\bigcup_{\alpha \in I} q_{\alpha}\left(\mathcal{T}^{\alpha}(k)\right) .
$$

Any point of $p(X(k)) \subset \mathbb{P}^{1}(k)$ either is in the image of one of the finitely many projections $C^{\alpha}(k) \rightarrow \mathbb{P}^{1}(k)(\alpha \in I)$, or is a zero or a pole of $f$.

Proof. Let $U \subset \mathbb{P}^{1}$ be the open set where $f$ is invertible, and let $V=X_{U}$ be its inverse image on $X$. The assumption on $f$ ensures that the divisor of this function, viewed as a divisor on $X$, is a double. Proposition 1.1 then shows that there exists a $\mathbb{Z} / 2$-torsor $\mathcal{T}$ over the whole of $X$ whose restriction to $V$ is given by the equation $f=u^{2}$. Since $X / k$ is proper, Proposition 1.2 ensures that the image of the evaluation map $\theta_{\mathcal{T}}$ is finite. We thus have the above finite decomposition. It remains to note that if $a \in k^{*}$ is a representative of $\alpha \in k^{*} / k^{* 2}$, the restriction $\mathcal{T}_{V}^{\alpha}$ of $\mathcal{T}^{\alpha}$ to $V$ is isomorphic to the $\mathbb{Z} / 2$-cover of $V$ defined by $f=a u^{2}$. In other words, $\mathcal{T}_{V}^{\alpha}$ is the fibre product of $V$ and $C_{U}^{\alpha}$ over $U$.

Remark 2.1.1. One may check that the normalisation of the fibre product $X \times_{\mathbb{P}^{1}} C_{\alpha}$ is unramified over $X$ at all codimension one points of $X$. It is thus unramified over the whole of $X$, hence is none other than $\mathcal{T}^{\alpha}$. This shows that the morphism $\mathcal{T}_{V}^{\alpha} \rightarrow C_{U}^{\alpha}$ extends to a morphism $\mathcal{T}^{\alpha} \rightarrow C^{\alpha}$, such that the composite $\mathcal{T}^{\alpha} \rightarrow C^{\alpha} \rightarrow \mathbb{P}_{k}^{1}$ coincides with the composite $\mathcal{T}^{\alpha} \rightarrow X \rightarrow \mathbb{P}_{k}^{1}$. Thus $p(X(k)) \subset \mathbb{P}^{1}(k)$ is contained in the union of the images of the projections $C_{\alpha}(k) \rightarrow \mathbb{P}^{1}(k)$. 
Corollary 2.2. Let $k$ be a field finitely generated over $\mathbb{Q}$. Let $p: X \rightarrow$ $\mathbb{P}_{k}^{1}$ be a standard fibration. Assume that the number $s$ of geometric double fibres is at least 6 . Then the $k$-points of $X$ are all located on finitely many fibres of $p$. In particular, they are not dense for the Zariski topology on $X$.

Proof. Recall that a geometric fibre is a fibre of the fibration $\bar{X} \rightarrow \mathbb{P}_{\bar{k}}^{1}$ obtained by going over to an algebraic closure $\bar{k}$ of $k$. To prove the corollary, we may replace the ground field $k$ by a finite extension. We may thus assume that there are $6 k$-rational points of $\mathbb{P}_{k}^{1}$ whose fibre is a double fibre. Let $f \in k\left(\mathbb{P}^{1}\right)$ be a rational function whose divisor on $\mathbb{P}^{1}$ is the sum of these 6 points, with multiplicity one, minus 6 times some other rational point. The curves $C^{\alpha}$ in Theorem 2.1 are double covers of $\mathbb{P}^{1}$ ramified in 6 points. They are thus of genus 2. By Faltings' theorem (quondam Mordell's conjecture) $([\mathrm{F}],[\mathrm{MD}])$ they have finitely many $k$-rational points. The result now follows from Theorem 2.1 .

At the price of some complication, one can reduce the 6 in the last corollary down to 5 .

Theorem 2.3. Let $k$ be a field finitely generated over $\mathbb{Q}$. Let $p: X \rightarrow \mathbb{P}_{k}^{1}$ be a standard fibration. Assume that there exist $r \geq 2$ geometric double fibres. Then there exist finitely many curves $C^{\alpha}$, each of them a smooth complete intersection of $r-2$ quadrics in $\mathbb{P}_{k}^{r-1}$, and surjective $k$-morphisms $p_{\alpha}: C^{\alpha} \rightarrow \mathbb{P}_{k}^{1}$, such that any k-point $M \in p(X(k))$ either has its fibre double or lies in the image of one of the finitely many projection maps $C^{\alpha}(k) \rightarrow$ $\mathbb{P}^{1}(k)$.

Proof. Let $\left\{P_{i}\right\}, i \in S$, be the set of closed points with double fibre, which we may assume all lie in $\mathbb{A}_{k}^{1} \subset \mathbb{P}_{k}^{1}$. Let $k_{i}$ be the residue field at $P_{i}$. We have $r=\sum_{i \in S} \operatorname{deg}_{k}\left(k_{i}\right)$. We may and will write $k_{i}=k[t] / N_{k_{i} / k}\left(t-e_{i}\right)$ with $e_{i} \in k_{i}$ such that $k_{i}=k\left(e_{i}\right)$.

Let $U \subset \mathbb{A}_{k}^{1}$ be the complement of $S$, and let $V=X_{U}=p^{-1}(U)$. Let

$$
V(k) \rightarrow\left(\prod_{i \in S} k_{i}^{*} / k_{i}^{* 2}\right) / \operatorname{Im} k^{*}
$$

be the map which sends a $k$-point $M$ with projection $t \in U(k) \subset \mathbb{A}^{1}(k)$ to the class of $\left\{t-e_{i}\right\}_{i \in S}$ in the quotient of the product $\prod_{i \in S} k_{i}^{*} / k_{i}^{* 2}$ by the image of the diagonal map $k^{*} \rightarrow \prod_{i \in S} k_{i}^{*} / k_{i}^{* 2}$.

We claim that the image of $V(k)$ under this map is finite.

There exists a regular, integral ring $O$, of finite type over $\mathbb{Z}$ (as an algebra), and an integral, regular, proper model $\widetilde{X} / \operatorname{Spec}(O)$, equipped with a $\operatorname{map} \pi: \widetilde{X} \rightarrow \mathbb{P}_{O}^{1}$, whose restriction to $\operatorname{Spec}(k)$ is $p: X \rightarrow \mathbb{P}_{k}^{1}$. For any point $P_{i}$, let $\widetilde{P}_{i}$ be the closure of $P_{i}$ in $\mathbb{P}_{O}^{1}$. Let $\widetilde{P}_{\infty}$ be the closure in $\mathbb{P}_{O}^{1}$ of the point at infinity of $\mathbb{P}_{k}^{1}$. Let $\Delta \subset \widetilde{X}$ be the divisor $\pi^{-1}\left(\widetilde{P}_{\infty}\right)$. 
By shrinking $O$, we may ensure that the following properties hold:

(a) For each $i$, the integral closure $O_{i}$ of $O$ in $k_{i}$ is regular and of finite type over $\mathbb{Z}$.

(b) The Picard group $\operatorname{Pic}(O)$ is trivial, and so is the Picard group of each of the $O_{i}$ (this uses the finiteness of class groups of number fields together with the finiteness theorems of Severi, Mordell and Néron).

(c) The unit group $O^{*}$ and each of the unit groups $O_{i}^{*}$ are finitely generated abelian groups.

(d) The projection map $\widetilde{X} \rightarrow \operatorname{Spec}(O)$ is proper. Thus for any $k$-rational point $M \in X(k)$ there exists an open set $U_{M} \subset \operatorname{Spec}(O)$ which contains all codimension one points, such that $M$ extends to a section $\widetilde{M} \in \widetilde{X}\left(U_{M}\right)$ over $U_{M}$. We shall refer to $\widetilde{M}$ as the closure of $M$.

(e) For any given $i$, the divisor of the rational function $t-e_{i}$ on $\widetilde{X} \times_{O} O_{i}$ is of the shape $2 \Delta_{i}-\Delta \times_{O} O_{i}$.

Let now $M \in V(k) \subset X(k)$ be an arbitrary point, and let $\widetilde{M} \in \widetilde{X}\left(U_{M}\right)$ be its closure. The divisor of $\left(t-e_{i}\right)(M) \in k_{i}^{*}$ on $\operatorname{Spec}\left(O_{i}\right)$ is

$$
2 \sigma_{M}^{*}\left(\Delta_{i}\right)-\sigma_{M}^{*}\left(\Delta \times{ }_{O} O_{i}\right)
$$

(since $O_{i}$ is regular, Cartier divisors and Weil divisors coincide, and are computed on codimension one points).

Using the commutative diagram of exact sequences:

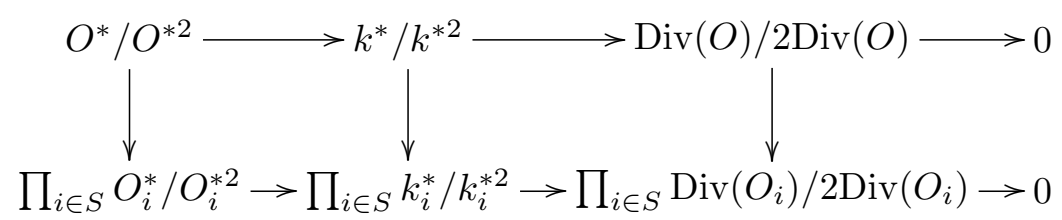

and the finite generation of the unit groups $O_{i}^{*}$, we conclude that the image of $\left\{\left(t-e_{i}\right)(M)\right\}_{i \in S}$ in the quotient $\left(\prod_{i \in S} k_{i}^{*} / k_{i}^{* 2}\right) / \operatorname{Im} k^{*}$ is finite.

Thus there exist finitely many elements $a=\left\{a_{i}\right\} \in \prod_{i \in S} k_{i}^{*}$ such that for any $k$-point of $V$, with projection $t \in \mathbb{A}^{1}(k)$, there exists one $a=\left\{a_{i}\right\}_{i \in S}$ such that the system of equations

$$
t-t_{i}=a_{i} v^{-1} \cdot \xi_{i}^{2} \neq 0 \quad(i \in S)
$$

has a solution with $v \in k^{*}$ and each $\xi_{i} \in k_{i}^{*}$. Viewing each $k_{i}$ as the set of $k$-points of an affine space over $k$ of dimension equal to the degree of $k_{i}$ over $k$, such a system of equations defines an algebraic $k$-variety $\mathcal{T}_{V}^{a}$. It is $k$-isomorphic to the open set $v \neq 0$ of the $k$-variety given by the system

$$
u-t_{i} v=a_{i} \xi_{i}^{2} \neq 0 \quad(i \in S) .
$$

The projection map to $\mathbb{A}_{k}^{1}$ now reads $t=u / v$. The variety given by the 
system

$$
u-t_{i} v=a_{i} \xi_{i}^{2} \quad(i \in S)
$$

is $k$-isomorphic, as one readily sees, to the cone over a curve $C^{a}$ which is a smooth complete intersection of $r-2$ quadrics in projective space $\mathbb{P}^{r-1}$ (smoothness is most easily seen by going over to the algebraic closure). Projection down to $\mathbb{A}_{k}^{1}$ is given by $t=u / v$, where $u$ and $v$ are both quadratic forms in the variables of $\mathbb{P}^{r-1}$. The map given by $t=u / v$ thus induces a dominant $k$-rational map, hence a morphism $C^{a} \rightarrow \mathbb{P}_{k}^{1}$, and the theorem follows.

Re mark 2.3.1. When $k$ is a number field, the finiteness theorems used in the proof are just the finiteness of the class groups of rings of integers and the finite generation of the unit groups. Since the ring of integers $O$ of a number field is of dimension one, points of $X(k)$ extend in this case to actual sections of $\widetilde{X} / \operatorname{Spec}(O)$.

R e mark 2.3.2. The proof we have given above is in the spirit of Weil's theory of distributions ("Arithmetic mirrors algebra", see [W.1], [W.2]). One may give a more abstract proof, in which one recognizes that the map

$$
V(k) \rightarrow\left(\prod_{i \in S} k_{i}^{*} / k_{i}^{* 2}\right) / \operatorname{Im} k^{*}
$$

studied above is the restriction to $V(k) \subset X(k)$ of the evaluation map $\theta_{\mathcal{T}}: X(k) \rightarrow H_{\text {et }}^{1}(k, S)$ attached to a torsor $\mathcal{T}$ over $X$ under a certain $k$ group of multiplicative type $S$. The interested reader is referred to [CT/S.2], Thm. 2.3.1, p. 420, and [CT/S.1], Prop. 2, p. 225.

Corollary 2.4. Let $k$ be a field finitely generated over $\mathbb{Q}$, and let $X / \mathbb{P}_{k}^{1}$ be a standard fibration. Assume that the number $r$ of geometric double fibres is at least 5 . Then the $k$-rational points of $X$ are not dense for the Zariski topology, but are concentrated on a finite number of fibres of $p$.

Proof. This is again an immediate consequence of Faltings' theorem ([F], [MD]). Indeed, a simple computation shows that for $r \geq 4$, the genus $g$ of a smooth complete intersection of $r-2$ quadrics in $\mathbb{P}^{r-1}$ is given by the formula

$$
2 g-2=2^{r-2}(r-4) .
$$

Thus $g \geq 5$ if $r \geq 5$. (For $r=4$, the genus is one, for $r=3$ or $r=2$, the genus is zero.)

3. Hyperelliptic surfaces. Pencils with double fibres have long been known in algebraic geometry. Most of the following proposition can be read off from [Sh], Chap. VII, [B], Chap. VI and [BPV], p. 148 and p. 189. 
Proposition 3.1. Let $k$ be a field, $\operatorname{char}(k) \neq 2$. Let $\bar{k}$ be an algebraic closure of $k$. Let $D / k$ be a curve of genus one equipped with a fixed point free involution $\varrho$. Let $C / k$ be a smooth, projective, geometrically integral curve equipped with a morphism $C \rightarrow \mathbb{P}_{k}^{1}$ of degree 2 . Let $\sigma$ denote the associated involution of $C$. Let $\tau$ be the involution on $Y=D \times{ }_{k} C$ which is given by the diagonal action $(M, N) \mapsto(\varrho(M), \sigma(N))$. Let $X$ be the quotient of $Y$ by this action. Then:

(1) The $k$-surface $X$ is smooth, projective and geometrically integral.

(2) The quotient map $Y \rightarrow X$ makes $Y$ into a $\mathbb{Z} / 2$-torsor over $X$.

(3) Let $D \rightarrow D_{1}$ be the unramified double cover defined by quotienting $D$ by the action of $\varrho$. Projection $D \times{ }_{k} C \rightarrow D$ induces a $k$-morphism $X \rightarrow D_{1}$ which is an Albanese map of $X$. Its geometric fibres are all smooth and isomorphic to $C \times \times_{k} \bar{k}$.

(4) Projection $D \times{ }_{k} C \rightarrow C$ induces a k-morphism $p: X \rightarrow \mathbb{P}_{k}^{1}$ which makes $X$ into a pencil of curves of genus one. The geometric fibres of $p$ are either smooth and isomorphic to $D \times{ }_{k} \bar{k}$ or they are double, the reduced fibre being then isomorphic to $D_{1} \times_{k} \bar{k}$. The double fibres occur exactly over the ramification points of the covering $C \rightarrow \mathbb{P}_{k}^{1}$.

(5) The surface $X$ is geometrically minimal, and has the following geometric invariants: $K_{X}^{2}=0, p_{g}=0, q=1$.

(6) The Kodaira dimension of $X$ is 1,0 or $-\infty$, depending on whether the genus of $C$ is at least 2 , equal to 1 , or equal to 0.

(7) There is no dominant rational map from $\bar{X}=X \times_{k} \bar{k}$ to a variety of general type.

Let us only comment on (7). By definition, a variety of dimension $d$ is of general type if its Kodaira dimension is equal to $d$ (see [BPV], p. 189). Suppose there is a dominant rational map from $\bar{X}$ to a surface of general type $Z$. Then there is a generically finite morphism from a blow-up $Y$ of $\bar{X}$ to $Z$. This implies that $Y$ is of general type ([BPV], I.(7.4), p. 23). By the birational invariance of plurigenera ([BPV], I.(9.1)(viii), p. 28), this implies that $\bar{X}$ is of general type, contradicting (6). It only remains to show that there is no dominant map from $\bar{X}$ to a curve of genus at least two. But this follows from $q=1$.

Note that if $D$ has a $k$-rational point, then one may use this point to make $D$ and $D_{1}$ into elliptic curves, and the map $D \rightarrow D_{1}$ into a $k$-isogeny whose kernel is a $k$-point of order 2 on the elliptic curve $D$.

We are now in a position to refute a conjecture attributed to J. Harris by D. Abramovich ([A]):

COROLlary 3.2. Let $k$ be a field finitely generated over $\mathbb{Q}$, let $D / k$ be a curve of genus one equipped with a fixed point free involution $\varrho$, let $C / k$ be a hyperelliptic curve of genus at least 2 , and let $\sigma$ denote the hyperelliptic 
involution on $C$. Let $\tau$ be the involution on $Y=D \times{ }_{k} C$ which is given by the diagonal action $(M, N) \mapsto(\varrho(M), \sigma(N))$. Let $X$ be the quotient of $Y$ by this action. Then:

(1) If $K / k$ is a field finitely generated over $k$, the set $X(K)$ is not Zariskidense in $X \times_{k} K$.

(2) There is no dominant rational map from $X \times_{k} \bar{k}$ to a variety of general type.

Proof. With notation as in the previous proposition, and as in Proposition 1.2 , let $\theta_{Y}: X(k) \rightarrow k^{*} / k^{* 2}$ be the evaluation map associated with the torsor $Y / X$. According to Proposition 1.2, $X(k)$ is the union of the projections of the $k$-points on finitely many $\mathbb{Z} / 2$-torsors $Y_{\alpha} / X$. Each $Y_{\alpha}$ becomes isomorphic to $Y=D \times C$ after a finite extension of the ground field $k$. By Faltings' result $([\mathrm{F}],[\mathrm{MD}])$, we conclude that the $k$-points on $Y_{\alpha}$ are not Zariski-dense. Thus the same holds for $X$. This proves (1). (One may also observe that according to Proposition 3.1(4), the fibration $X / \mathbb{P}_{k}^{1}$ has at least 6 geometric double fibres; one then applies Corollary 2.2.) As for (2), this has already appeared in the previous proposition.

Explicit affine equations for surfaces birational to $X$ as above will be given in the next section (see Examples 4.1.1 and 4.2.1, as well as Remark 4.2.2).

R e mark 3.2.1. Proposition 1.2 is a special case of a general theorem. Let $k$ be a number field, and let $Y \rightarrow X$ be a finite étale cover of smooth, projective, integral $k$-varieties. Assume that there is no finite field extension $L / k$ such that $Y(L)$ is Zariski-dense (such is the case if $Y$ dominates a curve of genus at least two $[\mathrm{F}])$. Then the same property holds for $X$. This is a direct consequence of Weil's "théorème des extensions non ramifiées" ([W.1], 1935). This result of Weil should be kept in mind when trying to amend the above mentioned conjecture.

4. Some explicit pencils of curves of genus one with double fibres. In this section, we describe some explicit pencils of curves of genus one with double fibres. The generic fibre of such a pencil is a curve of genus one over the field $K=k\left(\mathbb{P}^{1}\right)=k(t)$, without a $k(t)$-rational point. The simplest curves of genus one which do not obviously possess a rational point are smooth intersections of two quadrics in $\mathbb{P}^{3}$. The intersections of two quadrics which we describe arise naturally in classical descents on elliptic curves having at least one of their 2-torsion points rational over the ground field.

Let $k$ be a field, $\operatorname{char}(k)=0$. We shall consider surfaces $W$ given in affine space $\mathbb{A}_{k}^{4}$ with affine coordinates $x, y, z, t$ by a system of equations

$$
f(x, y, z, t)=0, \quad g(x, y, z, t)=0,
$$


where $f$ and $g$ are polynomials, $f$ and $g$ each of total degree 2 in the variables $x, y, z$. There is a natural projection $W \rightarrow \mathbb{A}_{k}^{1}$ given by the $t$-coordinate. The generic fibre of this projection is thus an intersection of two quadrics in affine space $\mathbb{A}_{k(t)}^{3}$. The assumptions we shall make on $f$ and $g$ will guarantee that this generic fibre is a geometrically integral curve whose projective model is a curve of genus one. We shall let $X$ be a smooth, projective, geometrically integral $k$-surface, $k$-birational to $W$, and equipped with a flat morphism $p: X \rightarrow \mathbb{P}_{k}^{1}$ birational to the projection $W \rightarrow \mathbb{A}_{k}^{1}$.

Proposition 4.1. Let $f(t)$ be a squarefree polynomial of even degree. Let $c(t), d(t)$ be nonzero polynomials, and assume that $f(t)$ and $c(t)-d(t)$ are coprime. Let $W$ be the affine variety defined by the system of equations

$$
x^{2}-c(t)=f(t) y^{2}, \quad x^{2}-d(t)=f(t) z^{2} .
$$

Let $p: X \rightarrow \mathbb{P}_{k}^{1}$ be a model as above. Then the divisor of $f(t)$ on $X$ is a double, and all the fibres of $p$ over the zeros of $f$ are double fibres. If $k$ is a field finitely generated over $\mathbb{Q}$, and the degree of $f$ is at least 6 , then the $k$-points of $W$ and $X$ are not dense for the Zariski topology.

Proof. Let $v$ be a discrete rank one valuation on the function field $k(X)$ of $X$. If it induces the trivial valuation on $k(t) \subset k(X)$, then certainly $v(f)=0$ is even. Assume $v$ induces a nontrivial valuation $w$ on $k(t)$. If $w$ is the valuation at infinity, $w(f)$ is then the opposite of the degree of $f$, hence is even, hence so is also $v(f)$. Now assume that $v$ is positive or zero on the ring $k[t]$. In the function field $k(X)$, we have the two equations

$$
\begin{aligned}
& x^{2}-c(t)=f(t) y^{2} \neq 0, \\
& x^{2}-d(t)=f(t) z^{2} \neq 0 .
\end{aligned}
$$

If $v(x)<0$, then $v\left(x^{2}-c(t)\right)=2 v(x)$ is even, hence $v(f)$ is even. Assume $v(x) \geq 0$. Now $v\left(x^{2}-c(t)\right) \geq 0$ and $v\left(x^{2}-d(t)\right) \geq 0$. If either $v\left(x^{2}-c(t)\right)=0$ and $v\left(x^{2}-d(t)\right)=0$, then (4.1.1) or (4.1.2) shows that $v(f)$ is even. If $v\left(x^{2}-c(t)\right)>0$ and $v\left(x^{2}-d(t)\right)>0$, then $v(c(t)-d(t))>0$. Since $f$ and $c-d$ are coprime, this implies $v(f)=0$. Corollary 2.2 now gives the last part of the proposition.

More generally, one may consider varieties $W$ given by a system of equations

$$
x^{2}-c(t)=f(t) y^{2}, \quad x^{2}-d(t)=g(t) z^{2},
$$

where one assumes $f(t) g(t) c(t) d(t)(c(t)-d(t)) \neq 0$. Let $p(t) \in k[t]$ be an irreducible polynomial. Let $v_{p}$ be the associated valuation on $k(t)$. Assume $v_{p}(f)=1, v_{p}(g)=1, v_{p}(c-d)=0$. Then the fibre of $X / \mathbb{P}_{k}^{1}$ over the closed point of $\mathbb{A}_{k}^{1}$ defined by $p=0$ is double. 
In $[\mathrm{CT} / \mathrm{Sk} / \mathrm{SwD} .1]$, we study pencils of curves of genus one given by equations

$$
x^{2}-c(t)=f(t) y^{2}, \quad x^{2}-d(t)=g(t) z^{2} .
$$

The algebraic conditions we impose precisely rule out the existence of $p(t)$ as above (namely $v_{p}(f)=1, v_{p}(g)=1, v_{p}(c-d)=0$ ). Taking some difficult but standard conjectures for granted, we then give sufficient conditions for $k$-rational points to be Zariski-dense on $X$.

In the situation of Proposition 4.1, the $\mathbb{Z} / 2$-coverings of Proposition 1.2 and Theorem 2.1 are easy to describe, at least birationally. Namely, letting $\alpha \in k^{*}$ denote a lift of the class $\alpha \in k^{*} / k^{* 2}$, the variety $\mathcal{T}^{\alpha}$ is $k$-birational to the variety given in affine space $\mathbb{A}_{k}^{5}$ with affine coordinates $x, Y, Z, t, u$ by the system

$$
x^{2}-c(t)=\alpha Y^{2}, \quad x^{2}-d(t)=\alpha Z^{2}, \quad f(t)=\alpha u^{2} .
$$

The system

$$
x^{2}-c(t)=\alpha Y^{2}, \quad x^{2}-d(t)=\alpha Z^{2}
$$

is a pencil of curves of genus one over $\mathbb{A}_{k}^{1}=\operatorname{Spec}(k[t])$, and $\mathcal{T}^{\alpha}$ is the pullback of this pencil under the projection of the hyperelliptic curve $f(t)=\alpha u^{2}$ down to $\mathbb{A}_{k}^{1}=\operatorname{Spec}(k[t])$.

Some particular cases are of interest.

EXAMPLE 4.1.1. Assume that $c(t)=c \in k$ and $d(t)=d \in k$ are constant $(c d(c-d) \neq 0)$. In this case Proposition 4.1 also holds for $f(t)$ separable of odd degree. The $k$-variety $\mathcal{T}^{\alpha}$ is $k$-birational to the product of a smooth projective curve $D^{\alpha} / k$ of genus one given in affine space $\mathbb{A}_{k}^{3}$ by the equation

$$
x^{2}-c=\alpha Y^{2}, \quad x^{2}-d=\alpha Z^{2}
$$

and a smooth projective hyperelliptic curve $C^{\alpha}$ given in affine coordinates by $f(t)=\alpha u^{2}$.

The involution $(x, Y, Z) \mapsto(x,-Y,-Z)$ induces a fixed point free involution $\varrho$ on $D^{\alpha}$. As for $C^{\alpha}$, it comes equipped with a hyperelliptic involution $\sigma$. One immediately checks that the diagonal involution $\tau$ on $D^{\alpha} \times{ }_{k} C^{\alpha}$ induces the birational involution of $\mathcal{T}^{\alpha}$ corresponding to the projection $\mathcal{T}^{\alpha} \rightarrow X$. Thus up to a birational equivalence, the case where $c$ and $d$ are constant leads to a special case of the situation described in Section 3.

An easy birational transformation turns the surface defined by the equation

$$
x^{2}-c=f(t) y^{2}, \quad x^{2}-d=f(t) z^{2}
$$

into the surface defined by

$$
Y^{2}=f(t)\left(x^{2}-c\right), \quad w^{2}=\left(x^{2}-c\right)\left(x^{2}-d\right) .
$$


Let $E / k$ be the smooth projective curve of genus one, with a $k$-rational point, defined by the affine equation $w^{2}=\left(x^{2}-c\right)\left(x^{2}-d\right)$. The above system of equations enables us to define a dominant $k$-rational map $X \rightarrow E$, the general geometric fibre of which is birational to the affine curve $Y^{2}=f(t)$. The quotient of $D^{\alpha}$ by $\varrho$ is $k$-isomorphic to $E$. The rational map $X \rightarrow E$ is $k$-birational to the projection $\left(C^{\alpha} \times{ }_{k} D^{\alpha}\right) / \tau \rightarrow D^{\alpha} /(\varrho) \simeq E$, which is an Albanese map (Section 3).

Note that we have a dominating map $D^{\alpha} \rightarrow E$, which can be made into an isogeny if $D^{\alpha}(k) \neq \emptyset$.

EXAmple 4.1.2. Another case of interest is the following. Assume that $f(t) c(t) d(t)(c(t)-d(t))$ is separable, each of the polynomials $f, c, d, c-d$ being of degree 2 . Then one checks that the relatively minimal fibration $X \rightarrow \mathbb{P}_{k}^{1}$ this leads to, when considered over an algebraic closure $\bar{k}$, has two double fibres and 6 other singular fibres, each of these being the union of two smooth conics in $\mathbb{P}^{3}$ intersecting transversally in two points. The $\mathbb{Z} / 2$-coverings $\mathcal{T}^{\alpha}$ this leads to (via $f(t)=\alpha u^{2}$ ) are surfaces fibred into curves of genus one over the curve of genus zero $C^{\alpha}$. This last fibration is equipped with exactly twelve singular geometric fibres, all of them of the type just described. One checks that $X$ is an Enriques surface, and that each $\mathcal{T}^{\alpha}$ is a $K 3$-surface. Such fibrations $\mathcal{T}^{\alpha} / C^{\alpha}$ are studied in [CT/Sk/SwD.1].

Here is another series of examples, which as a matter of fact is just a generalization of the previous series.

Proposition 4.2. Let $f, a, b, c \in k[t]$ be polynomials in $k[t]$ with $f$ separable of even degree and $f$ and ac coprime (hence in particular fac $\neq 0$ ). Assume $b^{2}-4 a c \neq 0$. Consider the surface $W$ given in affine space $\mathbb{A}_{k}^{4}$ with coordinates $t, x, y, z$ by the system

$$
\begin{gathered}
y^{2}=f(t) x, \\
z^{2}=f(t)\left(a(t) x^{2}+b(t) x+c(t)\right) .
\end{gathered}
$$

Let $p: X \rightarrow \mathbb{P}_{k}^{1}$ be a model as above. Then the divisor of $f(t)$ on $X$ is a double, and all the fibres of $p$ over the zeros of $f$ are double fibres. If $k$ is a field finitely generated over $\mathbb{Q}$, and the degree of $f$ is at least 6 , then the $k$-points of $W$ and $X$ are not dense for the Zariski topology.

Proof. To prove the proposition, it is enough to show that for any discrete rank one valuation $v$ on the field of functions $k(W)$ of $W$, the valuation $v(f)$ is even. We may restrict attention to valuations $v$ which induce a nontrivial valuation on the subfield $k(t)$ of $k(W)$.

If $v(t)<0$, then $v(f)$ is even since the degree of $f$ is even. Assume $v(t) \geq 0$. Then $v(f) \geq 0$. If $v(f)=0$, we are done. Assume $v(f)>0$. Then $v(a)=0$ and $v(c)=0$ by the coprimality assumption (since $v(t) \geq 0$, the 
valuation $v$ induces a valuation of $k(t)$ attached to a prime ideal of $k[t])$. If $v(x)<0$, then $v\left(a x^{2}+b x+c\right)=2 v(x)$ and (4.2.2) implies $v(f)$ even. If $v(x)>0$, then $v\left(a x^{2}+b x+c\right)=v(c)=0$ and (4.2.2) implies $v(f)$ even. If $v(x)=0$, then (4.2.1) implies $v(f)$ even. This completes the proof of the first statement. Corollary 2.2 now gives the last part of the proposition.

In the situation of Proposition 4.2, the $\mathbb{Z} / 2$-coverings of Proposition 1.2 and Theorem 2.1 are easy to describe, at least birationally. Namely, letting $\alpha \in k^{*}$ denote a lift of the class $\alpha \in k^{*} / k^{* 2}$, the variety $\mathcal{T}^{\alpha}$ is $k$-birational to the variety given in affine space $\mathbb{A}_{k}^{5}$ with affine coordinates $x, y, z, t, u$ by the system

$$
y^{2}=f(t) x, \quad z^{2}=f(t)\left(a(t) x^{2}+b(t) x+c(t)\right), \quad f(t)=\alpha u^{2} .
$$

Under the change of variables $Y=y / u, Z=z / u$, this is $k$-birational to the variety defined by

$$
Y^{2}=\alpha x \neq 0, \quad Z^{2}=\alpha\left(a(t) x^{2}+b(t) x+c(t)\right) \neq 0, \quad f(t)=\alpha u^{2} \neq 0 .
$$

Let $C^{\alpha}$ be the smooth projective curve given by the affine equation $f(t)=$ $\alpha u^{2}$. It comes equipped with the hyperelliptic map $C^{\alpha} \rightarrow \mathbb{P}_{k}^{1}$ extending the projection to $\mathbb{A}_{k}^{1}$ given by $(u, t) \mapsto t$. On the other hand, we have the variety defined by the system

$$
Y^{2}=\alpha x, \quad Z^{2}=\alpha\left(a(t) x^{2}+b(t) x+c(t)\right),
$$

which is a pencil of curves of genus one over $\mathbb{A}_{k}^{1}$. Thus the descent variety $\mathcal{T}^{\alpha}$ is described $k$-birationally as the pull-back to the smooth, projective, hyperelliptic curve $C^{\alpha}$, via its natural projection $C^{\alpha} \rightarrow \mathbb{P}_{k}^{1}$, of a $\mathbb{P}_{k}^{1}$-pencil of curves of genus one.

EXAMPLE 4.2.1. Just as before, some special cases are of interest. Assume $a(t)=a, b(t)=b, c(t)=c$ with $a, b, c \in k, a c \neq 0$ and $b^{2}-4 a c \neq 0$. In this case, Proposition 4.2 also holds for $f$ separable of odd degree. As above, we shall see that this special case is of the kind described in Section 3.

Any $\mathcal{T}^{\alpha}$ is $k$-birational to the product of the smooth, projective, hyperelliptic curve $C^{\alpha}$ with affine equation $f(t)=\alpha u^{2}$ and the smooth, projective curve of genus one $D^{\alpha} \subset \mathbb{P}^{3}$ with affine model in $\mathbb{A}_{k}^{3}$ given by the system of equations

$$
Y^{2}=\alpha x, \quad Z^{2}=\alpha\left(a x^{2}+b x+c\right) .
$$

This birational description will suffice for most purposes, but there may be some interest, at least from an aesthetic point of view, in giving nice smooth projective models for $X$ and the $\mathcal{T}^{\alpha}$. Models for the $\mathcal{T}^{\alpha}$ we already have, namely $C^{\alpha} \times_{k} D^{\alpha}$. There is a fixed point free involution $\varrho$ on $D^{\alpha}$, defined by $(x, Y, Z) \mapsto(x,-Y,-Z)$. We also have the hyperelliptic involution $\sigma$ on $C^{\alpha}$ given by $(t, u) \rightarrow(t,-u)$. We thus have a diagonal involution $\tau=(\varrho, \sigma)$ on $C^{\alpha} \times_{k} D^{\alpha}$, which we recall is $k$-birational to $\mathcal{T}_{\alpha}$. On the 
above equations, one immediately sees that the diagonal action on $C^{\alpha} \times_{k} D^{\alpha}$ reads as the nontrivial involution of the cover $\mathcal{T}_{\alpha} / X$. In particular, $X$ is $k$ birational to the quotient of $C^{\alpha} \times_{k} D^{\alpha}$ by the fixed point free action of $\varrho$ : this provides a beautiful model for $X$, equipped with a projection $X \rightarrow \mathbb{P}_{k}^{1}$.

Setting $w=z x / y$, we see that the surface given by

$$
y^{2}=f(t) x, \quad z^{2}=f(t)\left(a x^{2}+b x+c\right)
$$

(with $a, b, c \in k$ and $a c\left(b^{2}-4 a c\right) \neq 0$ ) is $k$-birational to the surface

$$
y^{2}=f(t) x, \quad w^{2}=x\left(a x^{2}+b x+c\right) .
$$

The last equation is the equation of an elliptic curve $E$ with a 2-torsion point. This gives a presentation of the surface $X$ as a fibration over the elliptic curve $E$, the generic fibre being a conic when $\operatorname{deg}(f(t))$ is 2 , a curve of genus one when $\operatorname{deg}(f(t))$ is 4 , a hyperelliptic curve in general. The projection $X \rightarrow E$ is an Albanese map (Section 3). We thus have dominating maps from the covering varieties $C^{\alpha} \times D^{\alpha}$ to $E$. Recall that $C^{\alpha}$ is given by $f(t)=\alpha u^{2}$ and $D^{\alpha}$ is the curve of genus one given by the system $z^{2}=\alpha\left(a x^{2}+b x+c\right)$, $y^{2}=\alpha x$. A direct computation shows that the map $C^{\alpha} \times D^{\alpha} \rightarrow E$ is given by $(x, y, z, t, u) \mapsto(w, x)$ with $w=z x / y$, hence this map factorizes through $D^{\alpha}$ :

$$
C^{\alpha} \times D^{\alpha} \rightarrow D^{\alpha} \rightarrow E .
$$

In particular, if $D^{\alpha}(k) \neq \emptyset$, then the map $D^{\alpha} \rightarrow E$ can made into a $k$ isogeny.

R e mark 4.2.2. Suppose $E / k$ is an elliptic curve equipped with a nontrivial point $A \in E(k)$ of order 2 . Let $\varrho$ be the translation by $A$. Let $C$ be a smooth, projective, geometrically integral curve equipped with a morphism $C \rightarrow \mathbb{P}_{k}^{1}$ of degree 2, with affine model $u^{2}=f(t)$, and let $\sigma$ be the hyperelliptic involution. Let $X$ be the quotient of $E \times{ }_{k} C$ by the diagonal involution $\tau$ (see Proposition 3.1). As a partial converse to what was done in 4.2.1, the reader will check that $X$ admits a birational model given in affine coordinates by a system $y^{2}=f(t) x, w^{2}=x\left(x^{2}+b x+c\right)$, with $b, c \in k^{*}$, $c\left(b^{2}-4 c\right) \neq 0$.

5. A conjecture of B. Mazur. In his papers [Maz.1] and [Maz.2], Mazur has proposed the following conjecture:

Conjecture 1. Let $X / \mathbb{Q}$ be a smooth integral variety. Assume that $X(\mathbb{Q})$ is dense for the Zariski topology on $X$. Then the topological closure of $X(\mathbb{Q})$ in $X(\mathbb{R})$ (for the euclidean topology) is a union of connected components of $X(\mathbb{R})$.

We shall now disprove this conjecture. We shall offer different types of counterexamples. The method followed is that suggested at the end of Section 1. For the rest of this section, we take $k=\mathbb{Q}$, the rational field. 
The simplest counterexamples are found among surfaces of the type described in Section 3, more precisely among the examples of such surfaces described in Section 4, Examples 4.1.1 and 4.2.1. As explained there, each variety $\mathcal{T}^{\alpha}$ is $\mathbb{Q}$-birational to $C^{\alpha} \times_{\mathbb{Q}} D^{\alpha}$, where $D^{\alpha}$, if it has a $\mathbb{Q}$-point, is $\mathbb{Q}$-isogenous to the elliptic curve $E$ (which is the Albanese variety of $X$ ). Since existence of a rational point and Zariski density of such points are birational invariants of smooth projective varieties, it is enough to establish properties (a), (b), (c) of Section 1 for the products $C^{\alpha} \times_{\mathbb{Q}} D^{\alpha}$.

We thus want:

(a) There is at least one $\alpha \in \mathbb{Q}^{*}$ with $\alpha>0$ and both $C^{\alpha}(\mathbb{Q})$ and $D^{\alpha}(\mathbb{Q})$ infinite.

(b) There exists at least one $\alpha \in \mathbb{Q}^{*}$ with $\alpha<0$ and $C^{\alpha}(\mathbb{Q})$ and $D^{\alpha}(\mathbb{Q})$ not empty.

(c) For any $\alpha \in \mathbb{Q}^{*}$ with $\alpha<0$, the set $C^{\alpha}(\mathbb{Q}) \times D^{\alpha}(\mathbb{Q})$ of $k$-points is not Zariski-dense in $C^{\alpha} \times_{\mathbb{Q}} D^{\alpha}$.

Since we have finite maps $D^{\alpha} \rightarrow E$ for any $\alpha$, in order to ensure these conditions, we need $E(\mathbb{Q})$ to be infinite. This then implies that any $D^{\alpha}(\mathbb{Q})$ which is not empty is infinite. We also need some $C^{\alpha}(\mathbb{Q})$ to be infinite, and some other $C^{\alpha}(\mathbb{Q})$ to be not empty and finite. Since $C^{\alpha}$ has affine equation $f(t)=\alpha u^{2}$, this forces us to take $f$ of degree 3 or 4 , i.e. the $C^{\alpha}$ 's must be curves of genus one.

We first develop Example 4.1.1.

Proposition 5.1. Let $f(t) \in \mathbb{Q}[t]$ be a separable polynomial of degree 3 or 4 . Assume that the curve of genus one $y^{2}=f(t)$ has infinitely many rational points, and that the curve $y^{2}=-f(t)$ has rational points but only finitely many. Let $r>0$ be an integer such that the elliptic curve $w^{2}=$ $u(u-1)\left(u+r^{2}\right)$ has positive rank over $\mathbb{Q}$. Let $W \subset \mathbb{A}_{\mathbb{Q}}^{4}$ be the surface defined by the system of equations

$$
x^{2}-r^{2}=f(t) y^{2}, \quad x^{2}-\left(r^{2}+1\right)=f(t) z^{2} .
$$

Let $X / \mathbb{Q}$ be a smooth projective model of $W / \mathbb{Q}$. Then the closure of $X(\mathbb{Q})$ in $X(\mathbb{R})$ contains one connected component of $X(\mathbb{R})$ but does not consist of a union of connected components of $X(\mathbb{R})$.

Pr o of. The divisor of the function $f(t)$ on $X$ is a double (Proposition 4.1 and remark at the beginning of 4.1.1). It thus defines a $\mathbb{Z} / 2$-torsor $\mathcal{T} / X$. Let us compute the image of the associated map $\theta: X(\mathbb{Q}) \rightarrow \mathbb{Q}^{*} / \mathbb{Q}^{* 2}$. For a given prime $p$, the image of the local map $X\left(\mathbb{Q}_{p}\right) \rightarrow \mathbb{Q}_{p}^{*} / \mathbb{Q}_{p}^{* 2}$ can be read off on any $U\left(\mathbb{Q}_{p}\right)$ for $U \subset X$ Zariski-open, not empty. We are thus free to argue with the variety defined by the system

$$
x^{2}-r^{2}=f(t) y^{2} \neq 0, \quad x^{2}-\left(r^{2}+1\right)=f(t) z^{2} \neq 0 .
$$


Let $(x, y, z, t)$ be a $\mathbb{Q}_{p}$-point on this variety. If $v_{p}(x)<0$, then $v_{p}\left(x^{2}-r^{2}\right)$ is even, hence also $v_{p}(f(t))$. Assume $v_{p}(x) \geq 0$. Now $v_{p}\left(x^{2}-r^{2}\right) \geq 0$ and $v_{p}\left(x^{2}-\left(r^{2}+1\right)\right) \geq 0$ and both cannot be strictly positive since the difference of their arguments is 1 . Thus either $v_{p}\left(x^{2}-r^{2}\right)=0$ or $v_{p}\left(x^{2}-\left(r^{2}+1\right)\right)=0$, and we deduce again that $v_{p}(f(t))$ is even.

Thus the image of $\theta_{\mathbb{Q}_{p}}$ in $\mathbb{Q}_{p}^{*} / \mathbb{Q}_{p}^{* 2}$ consists of elements with even valuation. Since this holds for any prime $p$, the image of $\theta_{\mathbb{Q}}$ is contained in $\pm 1 \in \mathbb{Q}^{*} / \mathbb{Q}^{* 2}$.

Now $\mathcal{T}=\mathcal{T}^{+1}$, which we simply write $\mathcal{T}^{+}$, is birational to the product of the smooth projective curve $C^{+}$with affine equation $y^{2}=f(t)$ (on which rational points are Zariski-dense by assumption) by the curve $D^{+}$given in affine space $\mathbb{A}_{\mathbb{Q}}^{3}$ by the system

$$
x^{2}-r^{2}=y^{2}, \quad x^{2}-\left(r^{2}+1\right)=z^{2} .
$$

This last curve has obvious rational points (at infinity), and it may easily be shown to be $\mathbb{Q}$-isogenous (and even $\mathbb{Q}$-isomorphic) to the curve $w^{2}=$ $u(u-1)\left(u+r^{2}\right)$. Our assumptions thus imply that $\mathbb{Q}$-points are Zariski-dense on $\mathcal{T}^{+}$.

As for $\mathcal{T}^{-1}$, denoted by $\mathcal{T}^{-}$, it is $\mathbb{Q}$-birational to the product of the smooth, projective curve $C^{-}$with affine equation $y^{2}=-f(t)$, on which rational points exist but are finitely many by assumption, and of the curve $D^{-}$given in affine space $\mathbb{A}_{\mathbb{Q}}^{3}$ by the system

$$
x^{2}-r^{2}=-y^{2}, \quad x^{2}-\left(r^{2}+1\right)=-z^{2} .
$$

This has the rational point $(x, y, z)=(r, 0,1)$.

EXAMPLE 5.1.1. For $f(t)$, we may take $f(t)=t^{3}-D$ with $D=-3$ (or any of $D=-5,7,-8,-9,-10, \ldots$, see Cassels' tables [Ca.1], p. 268/269). As for $r$, just take $r=3$. Observe that the elliptic curve $y^{2}=x(x-1)(x+9)$ has the rational point $(x, y)=(25 / 16,195 / 64)$, which by the Lutz-Nagell criterion $([\mathrm{K}]$, Thm. 5.1, p. 130) is not a torsion point. (The above point is just the double of the more obvious point $(x, y)=(9,36)$, as may be readily computed, see $[\mathrm{K}]$, p. 57 and p. 76.$)$

In a similar fashion, starting from Example 4.2.1, we obtain:

EXAMPLE 5.2. Let $W \subset \mathbb{A}_{\mathbb{Q}}^{4}$ be the surface defined by the system of equations

$$
y^{2}=\left(4 t^{4}+t^{2}-4\right) x, \quad z^{2}=\left(4 t^{4}+t^{2}-4\right)\left(x^{2}+4 x-1\right) .
$$

Let $X / \mathbb{Q}$ be a smooth projective model of $W / \mathbb{Q}$. Then $X(\mathbb{Q})$ is Zariski-dense in $X$. The space $X(\mathbb{R})$ has two connected components. In one of them rational points are dense for the real topology, in the other one their topological closure is contained in finitely many curves defined over $\mathbb{Q}$. 
The proof is similar to the previous one. We are indebted to Dr R. G. Pinch, whose computer calculations confirmed the relevant properties on the curves of genus one involved, which we had originally established by hand. Let $\mathcal{T} / X$ be the $\mathbb{Z} / 2$-torsor attached to the function $f(t)=4 t^{4}+t^{2}-4$, whose divisor is a double on $X$ (Proposition 4.2). One shows that the image of the associated map $X(\mathbb{Q}) \rightarrow \mathbb{Q}^{*} / \mathbb{Q}^{* 2}$ consists of \pm 1 .

The torsor $\mathcal{T}=\mathcal{T}^{+1}$, denoted by $\mathcal{T}^{+}$, is $\mathbb{Q}$-birational to the product of the elliptic curve $C^{+}$, with affine model $y^{2}=4 t^{4}+t^{2}-4$, which has infinitely many rational points, by the curve of genus one $D^{+}$given by the system

$$
y^{2}=x, \quad z^{2}=x^{2}+4 x-1 .
$$

This curve has $\mathbb{Q}$-points, and may thus be made into an elliptic curve isogenous to the elliptic curve $y^{2}=x\left(x^{2}+4 x-1\right)$, which has infinitely many rational points (it has a point with $x=1 / 4$, which is of infinite order by the Nagell-Lutz criterion). One easily checks that $C^{+}(\mathbb{R})$ and $D^{+}(\mathbb{R})$ are connected. Thus $C^{+}(\mathbb{Q}) \times D^{+}(\mathbb{Q})$ is dense in the connected space $C^{+}(\mathbb{R}) \times D^{+}(\mathbb{R})$. Since $\mathcal{T}^{+}$is smooth and projective and $\mathbb{Q}$-birational to $C^{+} \times_{\mathbb{Q}} D^{+}$, we also see that $\mathcal{T}^{+}(\mathbb{R})$ is connected, and that $\mathcal{T}^{+}(\mathbb{Q})$ is dense in $\mathcal{T}^{+}(\mathbb{R})$.

The torsor $\mathcal{T}^{-1}$, denoted by $\mathcal{T}^{-}$, is $\mathbb{Q}$-birational to the product of the elliptic curve $D^{-}$, with affine model $y^{2}=-\left(4 t^{4}+t^{2}-4\right)$, which has rational points but only finitely many, by the curve of genus one $C^{-}$given by the system

$$
y^{2}=-x, \quad z^{2}=-\left(x^{2}+4 x-1\right) .
$$

This last curve has rational points (e.g. $x=0$ ), hence may be made into an elliptic curve isogenous to the elliptic curve $y^{2}=x\left(x^{2}+4 x-1\right)$; it thus has infinitely many $\mathbb{Q}$-points. One easily checks that $C^{-}(\mathbb{R})$ and $D^{-}(\mathbb{R})$ are connected. Thus $C^{-}(\mathbb{R}) \times D^{-}(\mathbb{R})$ is connected, but the topological closure of the set of $\mathbb{Q}$-rational points on $C^{-} \times_{\mathbb{Q}} D^{-}$lies on finitely many $\mathbb{Q}$-rational curves. Since the smooth projective $\mathbb{Q}$-variety $\mathcal{T}^{-}$is $\mathbb{Q}$-birational to the variety $C^{-} \times_{\mathbb{Q}} D^{-}$, the same properties hold for $\mathcal{T}^{-}$. From the decompositions

$$
X(\mathbb{Q})=q_{+}\left(\mathcal{T}^{+}(\mathbb{Q})\right) \cup q_{-}\left(\mathcal{T}^{-}(\mathbb{Q})\right)
$$

and

$$
X(\mathbb{R})=q_{+}\left(\mathcal{T}^{+}(\mathbb{R})\right) \cup q_{-}\left(\mathcal{T}^{-}(\mathbb{R})\right)
$$

the result now follows.

Remark 5.2.1. Just as in Proposition 5.1, one may generate more examples by looking at surfaces given in affine space by a system

$$
y^{2}=f(t) x, \quad z^{2}=f(t)\left(x^{2}+r x-1\right),
$$

with $r \in \mathbb{Z}$ and $f(t) \in \mathbb{Q}[t]$ separable of degree 3 or 4 . Suitable examples of $f(t)$ are $f(t)=t^{3}-D$ for $D=-3,-5,7,-8,-9,-10, \ldots$ (see 5.1.1). 
R e mark 5.2.2. At the expense of some direct computations, the torsor theoretic language can be eliminated. Starting from the equations of $W$, the problem one faces is to construct a smooth projective model $X / \mathbb{Q}$, or at least a model $V / \mathbb{Q}$, such that the real points of $V$ are smooth and $V(\mathbb{R})$ is compact, so as to control the decomposition of $X(\mathbb{R})$ into connected components.

We now want to give an example of a different nature, which cannot be produced with the kind of surfaces considered in Section 3.

EXAmple 5.3. There exists a smooth projective surface $X / \mathbb{Q}$ such that $X(\mathbb{R})$ consists of two components, one in which $\mathbb{Q}$-rational points are dense and one which contains some but only finitely many $\mathbb{Q}$-rational points.

The surface $X$ will be a suitable smooth projective model of the surface $W$ given in affine space $\mathbb{A}_{k}^{4}$ with affine coordinates $x, y, z, \lambda$ by the system of equations

$$
\begin{aligned}
& y^{2}=\left(4 \lambda^{4}+\lambda^{2}-4\right) x, \\
& z^{2}=2\left(4 \lambda^{4}+\lambda^{2}-4\right)\left(\left(\lambda^{4}-2 \lambda^{2}+2\right) x^{2}+\left(\lambda^{2}+2\right) x-2\right) .
\end{aligned}
$$

Since we will want to say that there are only finitely many rational points in a given real component, it will be important to have a precise model (blowing up a rational point would ruin this property).

Start with the surface $Z_{1}$ given in $\mathbb{A}^{1} \times \mathbb{P}^{3}$ with coordinates $(\lambda ; x, y, z, t)$ by the system

$$
y^{2}=x t, \quad z^{2}=2\left(\left(\lambda^{4}-2 \lambda^{2}+2\right) x^{2}+\left(\lambda^{2}+2\right) x t-2 t^{2}\right) .
$$

Let the surface $Z_{2} \subset \mathbb{A}^{1} \times \mathbb{P}^{3}$ with coordinates $(\mu ; x, y, z, t)$ be defined by the system

$$
y^{2}=x t, \quad z^{2}=2\left(\left(1-2 \mu^{2}+2 \mu^{4}\right) x^{2}+\left(1+2 \mu^{2}\right) x t-2 t^{2}\right) .
$$

Letting $\mu=1 / \lambda$, one easily patches up these two surfaces into a proper surface $Z^{+}$equipped with a projection $Z^{+} \rightarrow \mathbb{P}_{\mathbb{Q}}^{1}$. One checks that the surface $Z^{+} / \mathbb{Q}$ is smooth (one may show it is the blow-up of a Del Pezzo surface of degree 2 in two points). The singular geometric fibres of $Z^{+} \rightarrow \mathbb{P}_{\mathbb{Q}}^{1}$ are located over the zeros of $\left(\lambda^{2}+2\right)^{2}+8\left(\lambda^{4}-2 \lambda^{2}+2\right)$ (the fibres are unions of two smooth conics in $\mathbb{P}^{3}$ meeting transversally in two points), and over the zeros of $\lambda^{4}-2 \lambda^{2}+2$ (the fibres are irreducible intersections of two quadrics in $\mathbb{P}^{3}$, with a simple node).

Let $C^{+} / \mathbb{Q}$ be the smooth projective curve with affine equation $w^{2}=$ $4 \lambda^{4}+\lambda^{2}-4$. Projection via the coordinate $\lambda$ makes this curve of genus one into a double cover $C^{+} \rightarrow \mathbb{P}_{\mathbb{Q}}^{1}$ which is ramified at the zeros of $4 \lambda^{4}+\lambda^{2}-4$. Note that these points are distinct from the points where $Z^{+} \rightarrow \mathbb{P}_{\mathbb{Q}}^{1}$ has nonsmooth fibres. Thus the fibre product $\mathcal{T}^{+}=Z^{+} \times_{\mathbb{P}_{\mathbb{Q}}^{1}} C^{+}$is a smooth 
and proper surface. On $Z_{1}$, there is an involution defined by $(\lambda ; x, y, z, t) \mapsto$ $(\lambda ; x,-y,-z, t)$. One checks that it extends to an involution on $Z^{+}$. We also have the hyperelliptic involution on $C^{+} / \mathbb{P}_{\mathbb{Q}}^{1}$. We thus get a diagonal involution $\sigma$ on $\mathcal{T}^{+}$. One checks that this involution has no fixed point. We then let $X$ be the quotient $\mathcal{T}^{+} / \sigma$. This is a smooth proper surface, equipped with a fibration $p: X \rightarrow \mathbb{P}_{\mathbb{Q}}^{1}$ (quotient of the fibration $\mathcal{T}^{+} \rightarrow C^{+}$).

One easily checks that the fibration $X / \mathbb{P}_{\mathbb{Q}}^{1}$ is $\mathbb{Q}$-birational to the fibration $W / \mathbb{A}_{\mathbb{Q}}^{1}$ described above. In particular, $\mathcal{T}^{+} / X$ is the $\mathbb{Z} / 2$-torsor attached to the rational function $f(\lambda)=4 \lambda^{4}+\lambda^{2}-4$, whose divisor is a double on $X$.

Factorization arguments over the rationals, of the kind we have seen several times, show that the image of the map $X(\mathbb{Q}) \rightarrow \mathbb{Q}^{*} / \mathbb{Q}^{* 2}$ associated with the torsor $\mathcal{T}^{+} / X$, and which on a suitable open set is given by the function $f$, has image contained in $\{ \pm 1\} \subset \mathbb{Q}^{*} / \mathbb{Q}^{* 2}$.

Thus we have the decompositions

$$
X(\mathbb{Q})=q_{+}\left(\mathcal{T}^{+}(\mathbb{Q})\right) \cup q_{-}\left(\mathcal{T}^{-}(\mathbb{Q})\right)
$$

and

$$
X(\mathbb{R})=q_{+}\left(\mathcal{T}^{+}(\mathbb{R})\right) \cup q_{-1}\left(\mathcal{T}^{-}(\mathbb{R})\right) .
$$

Here $\mathcal{T}^{-}$denotes the $\mathbb{Z} / 2$-torsor over $X$ which is given generically by the equation $f(\lambda)=-u^{2}$.

Let us first discuss $\mathcal{T}^{+}(\mathbb{Q})$ and $\mathcal{T}^{+}(\mathbb{R})$. Direct computation shows that the projection $Z^{+}(\mathbb{R}) \rightarrow \mathbb{P}^{1}(\mathbb{R})$ is surjective and that all the fibres are connected and topologically isomorphic to circles $S^{1}$. One also checks that $C^{+}(\mathbb{R})$ is connected. Therefore the fibres of $\mathcal{T}^{+}(\mathbb{R}) \rightarrow C^{+}(\mathbb{R})$ are connected and $\mathcal{T}^{+}(\mathbb{R})$ is connected.

The generic fibre $Z_{\eta}^{+}$of the fibration $Z^{+} \rightarrow \mathbb{P}_{\mathbb{Q}}^{1}$ is a smooth projective curve of genus one over $\mathbb{Q}(\lambda)$. We claim that $Z_{\eta}^{+}$has infinitely many $\mathbb{Q}(\lambda)$ points. There is a dominant $\mathbb{Q}(\lambda)$-map from $Z_{\eta}^{+}$to the elliptic curve with affine equation

$$
y^{2}=2 x\left(\left(\lambda^{4}-2 \lambda^{2}+2\right) x^{2}+\left(\lambda^{2}+2\right) x-2\right),
$$

hence $Z_{\eta}^{+}$, which has a rational point with $t=1, x=1 / \lambda^{2}$, can be viewed as an elliptic curve isogenous to the one we just wrote. Now this latter curve has the $\mathbb{Q}(\lambda)$-point $x=1 / \lambda^{2}, y=2 / \lambda^{3}$. But this curve has good reduction at $\lambda=0$. A classical argument then shows that torsion points of this curve with values in $\mathbb{Q}((\lambda))$ have coordinates in $\mathbb{Q}[[\lambda]]$ (see $[S]$, Chap. VII, Prop. 3.1). Thus the point $(x, y)=\left(1 / \lambda^{2}, 2 / \lambda^{3}\right)$ is of infinite order. Choosing an origin on $Z_{\eta}^{+}$now makes it into an elliptic curve with a point of infinite order. By a result of Silverman ([S], Appendix C, Thm. 20.3), for almost all points $M$ in $\mathbb{P}^{1}(\mathbb{Q})$, the fibre $Z_{M}^{+} / \mathbb{Q}$ is also of positive rank. Therefore for such $M$, the group $Z_{M}^{+}(\mathbb{Q})$ is topologically dense in $Z_{M}^{+}(\mathbb{R})$ (which is connected, as seen above). 
As we have mentioned in Example 5.2, the elliptic curve $C^{+} / \mathbb{Q}$ whose affine equation is $y^{2}=4 \lambda^{4}+\lambda^{2}-4$ has positive rank, and $C^{+}(\mathbb{R})$ is connected, so $C^{+}(\mathbb{Q})$ is topologically dense in $C^{+}(\mathbb{R})$. It now follows that $T^{+}(\mathbb{Q})$ is topologically dense in the connected space $T^{+}(\mathbb{R})$.

Let us now discuss $\mathcal{T}^{-}$. One may construct a smooth proper surface $Z^{-}$ just as above, starting with the surface defined in $\mathbb{A}^{1} \times \mathbb{P}^{3}$ with coordinates $(\lambda ; x, y, z, t)$ by the system

$$
y^{2}=-x t, \quad z^{2}=-2\left(\left(\lambda^{4}-2 \lambda^{2}+2\right) x^{2}+\left(\lambda^{2}+2\right) x t-2 t^{2}\right) .
$$

This surface comes equipped with a projection $Z^{-} \rightarrow \mathbb{P}_{\mathbb{Q}}^{1}$. Let $C^{-} / \mathbb{Q}$ be the smooth projective curve with affine equation $y^{2}=-\left(4 \lambda^{4}+\lambda^{2}-4\right)$. Projection via the coordinate $\lambda$ makes this curve of genus one into a double cover $C^{+} \rightarrow \mathbb{P}_{\mathbb{Q}}^{1}$ which is ramified at the zeros of $4 \lambda^{4}+\lambda^{2}-4$. These points are distinct from the points where $Z^{-} \rightarrow \mathbb{P}_{\mathbb{Q}}^{1}$ has nonsmooth fibres. Thus the fibre product $Z^{-} \times_{\mathbb{P}_{\mathbb{Q}}^{1}} C^{-}$is a smooth and proper surface. Just as above one produces a fixed point free involution on $Z^{-} \times_{\mathbb{P}_{\mathbb{Q}}^{1}} C^{-}$. Let $X^{\prime}$ be the quotient of this involution. One easily checks that it is $\mathbb{Q}$-birational to $X$, and that the fibration $Z^{-} \times_{\mathbb{P}_{\mathbb{Q}}^{1}} C^{-} \rightarrow X^{\prime}$ is $\mathbb{Q}$-birational to the fibration $\mathcal{T}^{-} \rightarrow X$. Going over to the algebraic closure of $\mathbb{Q}$, one finds that these birational equivalences are isomorphisms, hence they are isomorphisms over $\mathbb{Q}$. Thus $X^{\prime}=X$ and $T^{-}=Z^{-} \times_{\mathbb{P}_{\mathbb{Q}}^{1}} C^{-}$. The smooth projective curve $C^{-}$ with affine equation $y^{2}=-\left(4 \lambda^{4}+\lambda^{2}-4\right)$ has only the two rational points $(y, \lambda)=(2,0)$ and $(y, \lambda)=(-2,0)$. The fibre of $T^{-} / C^{-}$above either of these points is the curve of genus one given in $\mathbb{P}_{\mathbb{Q}}^{3}$ by the system of equations

$$
y^{2}=-x t, \quad z^{2}=-2\left(2 x^{2}+2 x t-2 t^{2}\right) .
$$

In affine coordinates, this is $z^{2}=-4\left(y^{4}-y^{2}-1\right)$. One checks that this curve has only finitely many rational points over $\mathbb{Q}$. Thus $\mathcal{T}^{-}(\mathbb{Q})$ is finite, and so is $q_{-}\left(\mathcal{T}^{-}(\mathbb{Q})\right)$.

Remark 5.3.1. One cannot produce examples as 5.3 starting from bielliptic surfaces as in Section 3 or Examples 4.1.1 and 4.2.1. It is an interesting question whether one could do this starting from Example 4.1.2. One however runs into the problem that we do not know a single example of a $K 3$-surface over a number field on which rational points exist without being Zariski-dense.

There are various ways of amending Mazur's conjecture. Two of them have been proposed by Mazur himself ([Maz.1] and [Maz.2], Conjectures 2 and 3). One may ask whether the topological closure of the set of $\mathbb{Q}$-rational points is always a semi-algebraic subset of $X(\mathbb{R})$. We would like to propose: 
ConjeCture 4 . Let $X$ be a smooth, integral variety over $\mathbb{Q}$, and let $U$ be a connected component of $X(\mathbb{R})$. If $W$ is the topological closure of $X(\mathbb{Q}) \cap U$ in $U$, then there is a Zariski-closed set $Y \subset X$ defined over $\mathbb{Q}$ such that $W$ is a (finite) union of connected components of $Y(\mathbb{R})$.

This would imply the weaker

Conjecture 5 . Let $X$ be a smooth, integral variety over $\mathbb{Q}$, and let $U$ be a connected component of $X(\mathbb{R})$. If $X(\mathbb{Q}) \cap U$ is Zariski-dense in $X$, then it is topologically dense in $U$.

\section{References}

[A] D. Abramovich, Lang maps and Harris's conjecture, Israel J. Math., to appear.

[BPV] W. Barth, C. Peters and A. Van de Ven, Compact Complex Surfaces, Ergeb. Math. Grenzgeb. (3) 4, Springer, 1984.

[B] A. Beauville, Surfaces algébriques complexes, Astérisque 54 (1978).

[Ca.1] J. W. S. Cassels, The rational solutions of the diophantine equation $Y^{2}=X^{3}-D$, Acta Math. 82 (1950), 243-273.

[Ca.2] -, Diophantine equations with special reference to elliptic curves, J. London Math. Soc. 41 (1966), 193-291.

[CT/S.1] J.-L. Colliot-Thélène et J.-J. Sansuc, La descente sur les variétés rationnelles, in: Journées de géométrie algébrique d'Angers, A. Beauville (éd.), Sijthoff and Noordhoff, Alphen aan den Rijn, 1980, 223-237.

[CT/S.2] —, - La descente sur les variétés rationnelles, II, Duke Math. J. 54 (1987), 375-492.

[CT/Sk/SwD.1] J.-L. Colliot-Thélène, A. N. Skorobogatov and Sir Peter Swinnerton-Dyer, Hasse principle for pencils of curves of genus one whose Jacobians have rational 2-division points, in preparation.

[CT/Sk/SwD.2] - - 一, 一, Rational points and zero-cycles on fibred varieties: Schinzel's hypothesis and Salberger's device, in preparation.

[CT/SwD] J.-L. Colliot-Thélène and Sir Peter Swinnerton-Dyer, Hasse principle and weak approximation for pencils of Severi-Brauer and similar varieties, J. Reine Angew. Math. 453 (1994), 49-112.

[F] G. Faltings, Complements to Mordell, in: Rational Points, Seminar Bonn/Wuppertal 1983/1984, G. Faltings, G. Wüstholz et al. (eds.), Aspekte der Math. E6, Vieweg, 1984, 203-227.

[K] A. W. Knapp, Elliptic Curves, Princeton University Press, 1992.

[MD] M. Martin-Deschamps, La construction de Kodaira-Parshin, Astérisque 127 (1985), 256-272.

[Maz.1] B. Mazur, The topology of rational points, Experiment. Math. 1 (1992), 35-45.

[Maz.2] -, Speculations about the topology of rational points: an up-date, Astérisque 228 (1995), 165-181.

[R] D. E. Rohrlich, Variation of the root number in families of elliptic curves, Compositio Math. 87 (1993), 119-151. 
[Sh] I. R. Shafarevich et al., Algebraic surfaces, Proc. Steklov Inst. Math. 75 (1967).

[S] J. H. Silverman, The Arithmetic of Elliptic Curves, Grad. Texts in Math. 106, Springer, 1986.

[Sk] A. N. Skorobogatov, Descent on fibrations over the projective line, Amer. J. Math. 118 (1996), 905-923.

[SwD] Sir Peter Swinnerton-Dyer, Rational points on certain intersections of two quadrics, in: Abelian Varieties, Proc. Conf. Egloffstein, W. Barth, K. Hulek and H. Lange (eds.), de Gruyter, Berlin, 1995, 273-292.

[W.1] A. Weil, Arithmétique et géométrie sur les variétés algébriques, in: Actualités Sci. Indust. 206, Hermann, Paris, 1935, 3-16; reprinted in: Oeuvres Scientifiques, Vol. I, Springer, 1980, 87-100.

[W.2] —, Arithmetic on algebraic varieties, Ann. of Math. 53 (1951), 412-444.

C.N.R.S., URA D0752

Mathématiques

Bâtiment 425

Université de Paris-Sud

F-91405 Orsay, France

E-mail: Jean-Louis.Colliot-Thelene@math.u-psud.fr

Institute for Problems of Information Transmission

Russian Academy of Sciences

19, Bolshoi Karetnyi

Moscow 101447, Russia

Laboratoire de mathématiques discrètes, C.N.R.S., UPR 9016

Equipe "Arithmétique et théorie de l'information"

Luminy Case 930

F-13288 Marseille Cédex 9, France

E-mail: skoro@ippi.ac.msk.su

Isaac Newton Institute

20 Clarkson Road

Cambridge CB3 0EH, Great Britain

E-mail: hpfs100@newton.cam.ac.uk 